\title{
Predicción orientada a impactos en el proyecto piloto de presencia de predictores en Centros de Control Aéreo
}

https://doi.org/10.31978/639-19-010-0.687

\author{
Juan de Dios Soriano Romero ${ }^{1}$ (jsorianor@aemet.es) \\ Ángel Alcázar Izquierdo² (aalcazari@aemet.es)
}

${ }^{1}$ AEMET / Delegación Territorial en Andalucía, Ceuta y Melilla

${ }^{2}$ AEMET / Dirección de Planificación, Estrategia y Desarrollo Comercial

\begin{abstract}
RESUMEN
La importancia de la meteorología para la navegación aérea es máxima, tanto para la seguridad de los tráficos como para la eficiencia de la gestión de los mismos. El aumento de la densidad de tráfico aéreo y el objetivo de superar niveles de seguridad ya de por sí muy elevados hace que los servicios de tráfico aéreo (siglas en inglés ATS) reclamen una atención cada vez más específica y adaptada a sus necesidades.
\end{abstract}

Actualmente, a petición de ENAIRE (principal proveedor de servicios de navegación aérea y de información aeronáutica en España), AEMET desarrolla un proyecto para la futura implantación de unidades de asesoría meteorológica en los centros de control de área, y en el seno del mismo viene funcionando con carácter preoperativo un servicio piloto en los centros de control de Torrejón, Gavá y Sevilla. Una de las necesidades detectadas en el servicio piloto ha sido la definición de nuevos productos que faciliten la toma de decisiones operativas por parte de los actores implicados. Los productos oficiales del catálogo OACI (Organización de Aviación Civil Internacional) no bastan. Se necesitan predicciones orientadas al impacto de los fenómenos meteorológicos en la operatividad de los centros de control, elaboradas con la antelación suficiente para la puesta en marcha de las medidas de mitigación de riesgos u optimización de flujos que sea necesario implementar. Un mismo fenómeno puede tener impactos muy diferentes dependiendo del lugar y del momento de ocurrencia. Y para la toma de decisiones es importante asimismo tener información de la fiabilidad de la predicción, por lo que esta debe tener un carácter probabilista.

En esa línea se avanza en el servicio piloto, actualmente en marcha, hacia productos basados en matrices de riesgo que tengan en cuenta el impacto de los fenómenos meteorológicos y su probabilidad de ocurrencia. Mientras tanto, se elaboran ya productos de texto intermedios en línea similar.

PALABRAS CLAVE: Centro de Control Aéreo; predicción orientada a impactos; TMA; ATS; matriz de impactos.

\section{INTRODUCCIÓN}

El apoyo a la aeronáutica ha sido siempre uno de los objetos principales de atención de la meteorología operativa. Muchos servicios meteorológicos nacionales han surgido inicialmente como herramientas de apoyo a la navegación aérea. Algunos están incluso encuadrados y dependen estructuralmente de las agencias y organismos encargados del control del tráfico aéreo en sus respectivos países. 
La OACI y la OMM (Organización Meteorológica Mundial) mantienen un completo catálogo de productos meteorológicos aeronáuticos que elaboran los distintos servicios meteorológicos y que cubren gran parte de las necesidades en materia de planificación de vuelos, en aras del mantenimiento de elevadísimos niveles de seguridad junto con la máxima eficacia y eficiencia en el tráfico aéreo mundial. Así, se elaboran de acuerdo con la normativa internacional observaciones y pronósticos de aeródromo, y mensajes y mapas de fenómenos significativos en ruta, además de información general sobre el estado de la atmósfera a distintos niveles en la vertical, desde vientos a temperaturas, pasando por humedad y otros parámetros, o incluso pronósticos especializados sobre la evolución prevista de ciclones tropicales o nubes de ceniza volcánica.

AEMET, en su evolución histórica desde los tiempos del SMN (Servicio Meteorológico Nacional) y del INM (Instituto Nacional de Meteorología), ha tenido siempre como uno de sus pilares de actuación el apoyo a la navegación aérea, manteniendo oficinas de vigilancia meteorológica (OVM) encargadas de pronósticos de ruta, oficinas de aeródromo (OMA) y oficinas encargadas de pronósticos de aeródromo, que vienen elaborando y difundiendo los productos del catálogo OACI.

En los últimos tiempos, el aumento del número de vuelos y la necesidad de mantener los estándares de seguridad y de puntualidad de los mismos han venido demandando un incremento del apoyo meteorológico, particularmente en el ámbito del control del tráfico aéreo. La incidencia, por ejemplo, de una tormenta en un área especialmente sensible, como un aeropuerto de gran densidad de tráfico o en su área de aproximación es muy alta y los productos tradicionalmente disponibles deben ser complementados para que la reacción operativa de los actores responsables del control aéreo sea óptima. Así se viene haciendo en los últimos años por parte de AEMET, elaborando nuevos productos, aumentando el conocimiento de los mismos o facilitando el contacto entre los responsables del control aéreo y los predictores meteorológicos. Asimismo, a petición de ENAIRE, se está desarrollando un proyecto para implementar próximamente unidades de asesoría meteorológica en los centros de control aéreo. En el seno de dicho proyecto se desarrolla una experiencia piloto, a modo de servicio preoperativo, con carácter más o menos continuo en varios de los centros de control de ENAIRE. Esta experiencia piloto está sirviendo entre otras cosas para identificar necesidades y diseñar el servicio a prestar por parte de las futuras unidades operativas.

Una de las necesidades más importantes detectadas es la elaboración de predicciones orientadas a impacto que faciliten la toma de decisiones operativas por parte de los responsables del servicio ATS, particularmente a nivel de TMA (siglas de Terminal Manouvering Area), áreas de espacio controlado alrededor de uno o varios aeropuertos importantes, donde confluye la aproximación con una serie de aerovías. Es en el TMA donde mayor complejidad presenta el control y donde la adecuación de los pronósticos meteorológicos disponibles es más escasa.

En esta ponencia se describirán las características que deberán tener los pronósticos de impacto a elaborar por las futuras unidades y los productos intermedios de predicción orientada a impactos que se desarrollan en la experiencia piloto. Previamente se describirán de forma somera la operatividad del control organizado del tráfico aéreo y la incidencia de la meteorología en el mismo.

\section{EL TRÁFICO AÉREO Y EL ESPACIO AÉREO: CENTROS DE CONTROL}

En paralelo a la aparición y el crecimiento del tráfico aéreo se vino desarrollando el control organizado del mismo. Inicialmente se hizo necesario en aeropuertos para evitar colisiones entre aeronaves y su necesidad se fue extendiendo a las rutas. El incremento en la densidad del tráfico ha ido llevando a una organización cada vez más compleja del control. Y a la que era la necesidad inicial, el mantenimiento de la seguridad de los tráficos, se fue uniendo pronto la necesidad de maximizar la eficacia y la eficiencia de los mismos, es decir permitir el mayor número de vuelos posible minimizando las demoras y cancelaciones. 
El espacio aéreo se organiza y subdivide a la vez que se organiza el control: regiones de información de vuelo, aerovías, sectores de vuelo, TMA, CTA (siglas de Controlled Traffic Area), zonas de vuelo prohibido, restringido o peligroso (en muchos casos por actividades de Defensa)... A la vez, cada fase del vuelo tiene asignado un componente del control aéreo. Así, los despegues y aterrizajes y las operaciones en pista son autorizados por el control de torre. Una vez en el aire (o a punto de iniciar el descenso) los tráficos pasan al control de aproximación. Durante el tránsito a niveles de crucero son llevados por el control de ruta. El control de ruta y el de aproximación corresponden a los centros de control de tránsito aéreo, aunque a veces la aproximación se delega a instalaciones locales.

Cada sector de vuelo es competencia de una pareja de controladores, cuya principal misión es mantener la separación entre aeronaves por encima de los mínimos que marque la normativa aplicable en cada caso. La capacidad de un sector es limitada. En el caso de un aeropuerto o de su aproximación, la disponibilidad de pistas o incluso de aparcamientos ya establece un límite sobre el número de aviones que pueden transitar. Además, en cualquier sector, el controlador debe establecer contacto con los aviones de su sector al menos dos veces, cuando entran y cuando salen de él. Eso establece otro límite, el número máximo de aeronaves que puede controlar un controlador. El número de comunicaciones se incrementa en caso de mala meteorología u otras incidencias (autorizaciones de desvíos, organización de esperas, comunicación de datos...), por lo que puede ser necesario rebajar la capacidad de los sectores afectados. La carga de trabajo incide también en el estrés, así que no debe exceder unos límites para asegurar que las decisiones operativas se toman de forma óptima.

El máximo responsable en el centro de control es el jefe de sala. Es quien toma todas las decisiones operativas más allá de las propias de los controladores, decisiones que pueden ir desde organizar sectores hasta establecer regulaciones (limitar capacidad), incluso de los aeropuertos o dependencias de aproximación con competencias delegadas. También puede poner en marcha los procedimientos de emergencia o de desvíos masivos. Para desarrollar su trabajo se apoya en otros actores del centro de control: el técnico de control de afluencia (responsable del análisis y previsión de flujos aéreos y de recomendar actuaciones en relación a la capacidad de sectores) y los supervisores (coordinadores de un equipo de controladores). Las decisiones del jefe de sala van encaminadas a la doble misión del control: mantener la seguridad y minimizar los retrasos. Una medida regulatoria siempre implica retrasos o cancelaciones, pero no tomarla puede tener consecuencias peores, tanto en cuanto a la seguridad como a la puntualidad. Debe tener, por tanto, una información lo más ajustada posible tanto de los tráficos previstos como de las posibles incidencias, incluidas las meteorológicas, por supuesto.

Las medidas regulatorias serán más efectivas si se toman con la antelación suficiente. Lo ideal es que se apliquen en la fase pretáctica (el día antes) o táctica (antes del despegue de los aviones). Es preferible siempre tener los aviones en tierra a tenerlos en el aire sin poder aterrizar. Aun así, también a los aviones en vuelo se pueden aplicar medidas que palíen los efectos de una incidencia sobrevenida. La antelación ideal depende de cada centro de control, según sea el tiempo medio de vuelo de los tráficos a controlar. En España, con gran parte de los flujos precedentes de Europa, la antelación debe ser de unas tres horas en el centro de control de Sevilla, de entre dos y tres horas en los de Torrejón, Gavá y Palma y casi de cinco horas en el de Gran Canaria.

Por último, una cuestión importante es que las decisiones se toman en función del impacto de la incidencia prevista. El tráfico no es el mismo en todos los puntos del espacio aéreo ni es homogéneo en el tiempo, por lo que una misma incidencia puede tener distintos impactos. Y es importante que la información que reciba el jefe de sala ya esté orientada al impacto en la operatividad, para facilitar su capacidad de reacción. No es más útil la información más amplia si después tiene que ser procesada por el mismo jefe de sala para determinar los posibles impactos. Ampliaremos esta cuestión en el siguiente apartado. 


\section{LA METEOROLOGÍA Y EL CONTROL AÉREO}

La meteorología es el principal causante de retrasos en la navegación aérea (fig. 1), y a veces también está en el origen de accidentes o incidentes. En cualquier caso y para lo que concierne a esta ponencia, basta que un fenómeno meteorológico pueda producir un aumento de las comunicaciones entre controladores y pilotos para que tenga incidencia en la operatividad del control. La meteorología incide en todas las fases del vuelo, desde el despegue al aterrizaje. No solo hay que considerar las incidencias que puedan afectar a los tráficos en ruta o en evolu-

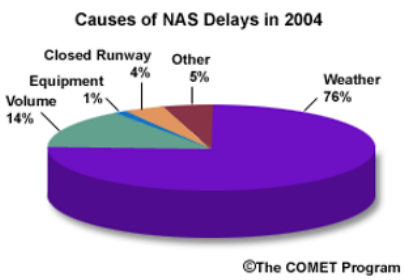

Rankings of Impact to the NAS by Significant Weather Elements

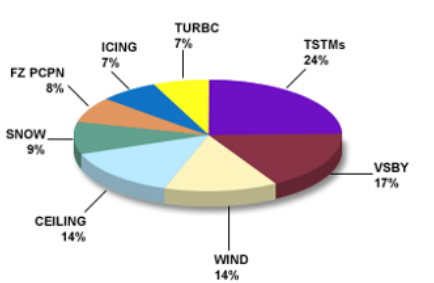

NOAA / NWS

Figura 1. Causas de retrasos en el espacio aéreo de EEUU y desglose por fenómenos meteorológicos.

Fuente: COMET (Cooperative Program for Operational Meteorology, Education and Training). ción, las incidencias en aeropuertos principales terminan afectando también a la operatividad del centro de control, bien directamente porque el jefe de sala tenga que tomar medidas de reducción de capacidad del aeródromo, o bien indirectamente porque implique actuaciones o comunicaciones con los tráficos en aproximación o incluso en ruta.

Son muchos los fenómenos meteorológicos a considerar, dependiendo de la fase de vuelo de que se trate (fig. 2). Algunos no están relacionados directamente con lo que podría considerarse tiempo adverso o severo. Basta un cambio de dirección del viento en superficie en un aeropuerto principal para que la redistribución subsiguiente de los flujos en la aproximación pueda provocar una situación complicada. O la existencia de corrientes en chorro en niveles altos puede implicar la necesidad de regulación de capacidad de algunos sectores, que pueden verse saturados, pues muchas aeronaves intentarán aprovechar los vientos más favorables transitando por ellos.

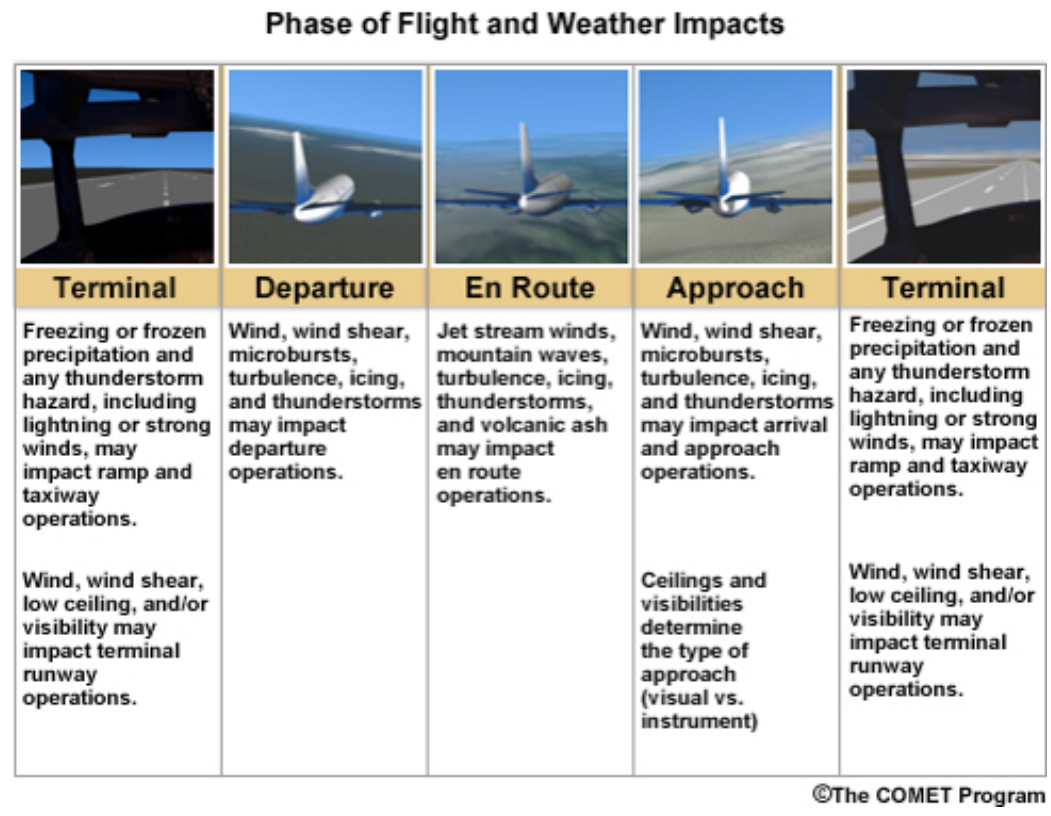

Figura 2.

Fases del vuelo y fenómenos meteorológicos con impacto. Fuente: COMET.

No obstante lo anterior, hay un fenómeno que sobresale sobre los demás en cuanto a su incidencia en la operatividad de los centros de control aéreo: las tormentas. Su importancia es doble. Suelen llevar asociados algunos de los factores meteorológicos más importantes como causa de retrasos o incidentes: vientos fuertes en superficie, contaminación de pista por agua, cizalladura, turbulencia, engelamiento... Pero, además, deben ser considerados como auténticos ocupantes del espacio aéreo, limitan su disponibilidad y reducen directamente su capacidad, además de provocar desvíos y complicar la gestión de las rutas. 
Como se dijo en el apartado anterior, el tráfico aéreo no es homogéneo ni en el tiempo ni en el espacio, Hay zonas o puntos, en el área de responsabilidad de cada centro de control, donde una incidencia cualquiera puede tener un impacto mucho mayor que en otras. Una tormenta fuerte y persistente en una zona fuera de aerovías o TMA puede pasar desapercibida en la operatividad, mientras una situación de cizalladura sobre un aeropuerto principal puede hacer que se active el procedimiento de desvíos masivos.

La información meteorológica que proporcionan los productos del catálogo OACI es amplia y de calidad. No obstante, debe ser complementada para permitir una actuación ágil y eficaz por parte de los actores del centro de control. Los productos de aeródromo son precisos, pero no pueden aplicarse en el entorno del aeropuerto ni en niveles superiores y no incluyen directamente predicciones de algunos fenómenos importantes como la cizalladura. Los productos de área no tienen la suficiente resolución espacial o temporal que sería conveniente para la toma de decisiones regulatorias, o tienen escaso adelanto, como los SIGMET (Significant Meteorological Information —-mensajes meteorológicos sobre fenómenos peligrosos para la aviación-). En todo caso, no son productos orientados al impacto de los fenómenos meteorológicos en la operatividad de los centros de control. Particularmente, a nivel de TMA, donde mayor es la complejidad del control aéreo, es donde se observa una mayor necesidad de nuevos productos que ayuden a la toma de decisiones operativas.

Es necesario, por tanto, proporcionar a ENAIRE como suministrador de servicios de tránsito aéreo (en siglas, ATS), predicciones orientadas a impacto, ajustadas a sus necesidades, teniendo en cuenta lugares y momentos clave y con umbrales según la incidencia de cada fenómeno en la operatividad. Estas predicciones podrán de este modo servir como entradas en los procedimientos de ENAIRE relacionados con la meteorología y facilitar la toma adecuada de decisiones por parte de los jefes de sala.

En esa línea se trabaja actualmente. AEMET suministra diversos productos automáticos (briefing para jefes de sala, descargas eléctricas previstas en TMA...) para mejorar la disponibilidad de información útil. Asimismo, a petición de ENAIRE está en marcha un proyecto para implementar unidades de asesoría meteorológica en los centros de control. En el seno de dicho proyecto se lleva a cabo una experiencia piloto a modo de servicio preoperativo que está sirviendo para identificar necesidades y definir el servicio a prestar por las futuras unidades. Una de las principales actividades es el desarrollo de predicciones orientadas a impacto.

\section{PREDICCIÓN ORIENTADA A IMPACTOS EN EL PROYECTO PILOTO DE PRESENCIA EN CENTROS DE CONTROL}

Como se ha dicho en el apartado anterior, se está desarrollando un proyecto piloto con las características de un servicio preoperativo de asesoría meteorológica en varios centros de control de ENAIRE. Una vez identificadas las necesidades de información meteorológica se ha definido un servicio basado en tres actividades principales:

- Briefing meteorológico, en la sala de control, para el jefe de sala y el resto de los actores principales del centro (técnico de control de afluencia, supervisores...), para suministrar la información más relevante para la operatividad, asegurando la unicidad y homogeneidad de la misma.

- Asesoría experta continua para el jefe de sala, de carácter proactivo, incluyendo vigilancia continua de la evolución de la situación meteorológica.

\section{- Productos meteorológicos de impacto.}

Como se expuso anteriormente, los productos OACI de predicción aeronáutica de área tienen escasa precisión espaciotemporal (mapas de tiempo significativo - SIGWX) o escaso adelanto y/o alcance (SIGMET) teniendo en cuenta las necesidades de los Centros de Control, y los productos de aeródromo son precisos y adelantados, pero están centrados en superficie y sobre el aeropuerto, no son útiles para la 
aproximación, por ejemplo. Además, el mismo fenómeno meteorológico puede tener distintos impactos, dependiendo de la zona, la hora de ocurrencia o determinadas características (extensión vertical y horizontal, por ejemplo), en función siempre de las características del tráfico aéreo. Y el nivel de riesgo (y por tanto la decisión operativa) debe depender también del nivel de confianza del pronóstico.

Teniendo en cuenta todo esto, los productos a elaborar por las futuras unidades de AEMET en centros de control deberían reunir las siguientes características:

- deben responder a las necesidades operativas del centro de control;

- deben cubrir el hueco existente de productos de predicción meteorológica aeronáutica, sobre todo a nivel de TMA;

- deben permitir la integración en los protocolos de actuación de ENAIRE, es decir, deben estar orientados a impactos;

- los productos de cada centro de control deben ser de características similares, pero adaptados a las necesidades particulares de cada uno de ellos;

- deben ser absolutamente compatibles con el resto de productos de predicción de AEMET, lo que implica dar la máxima importancia a la coordinación.

A continuación, se exponen las características básicas que podrían tener los futuros productos de impacto meteorológico a elaborar por las nuevas unidades, basados en matrices de impacto, así como los productos orientados a impacto que se elaboran actualmente en el seno del proyecto piloto.

\subsection{Productos basados en matrices de impacto}

Las matrices de impacto son de uso común en numerosos ámbitos: Defensa, negocios... y también en Meteorología. Sirven para asesorar la decisión sobre las medidas a tomar en función del nivel de riesgo y de la probabilidad de ocurrencia de una circunstancia determinada, en nuestro caso un fenómeno meteorológico. Es fundamental definir de forma adecuada los umbrales para los niveles de riesgo, así como la escala de probabilidades. También es clave la asignación de colores a las celdas de la matriz, ya que cada color implicará un determinado nivel de actuación. Pueden definirse distintos niveles de riesgo o incluso colores de celda distintos dependiendo del fenómeno meteorológico, el lugar o la hora del día. Un ejemplo de matriz de impactos puede verse en la figura 3.

El diseño de los productos basados en matrices de impacto implica, por tanto, varios procesos:

- Definición de puntos y zonas clave y fenómenos con impacto.

\section{Matriz de impacto por tormentas Aproximación a LEMG}

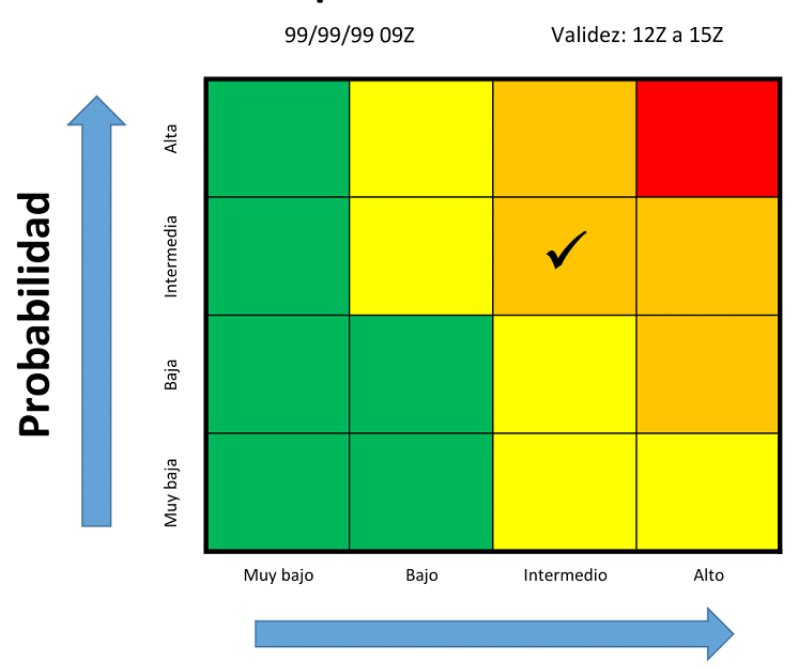

Impacto

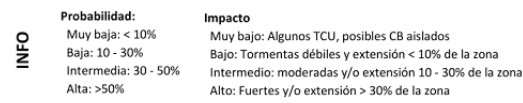

Figura 3. Ejemplo ficticio de matriz de impactos por tormentas en la aproximación al aeropuerto de Málaga.

- Definición de umbrales de riesgo para distintos fenómenos y horarios. Un ejemplo tentativo puede verse en la figura 4.

- Definición de la escala de probabilidades.

- Asignación de colores a las celdas en función del nivel de riesgo y la probabilidad de ocurrencia, de modo que cada color implica un nivel de actuación (ejemplo tentativo en tabla). 
- Definición de frecuencia, adelanto y alcance de los pronósticos.

- Definición y diseño de la salida gráfica.

Se elaborarían pronósticos para cada fenómeno y punto de interés, con el plazo de validez y el adelanto que sea necesario para la operatividad del centro de control. Estos pronósticos, después, se podrían resumir en un producto combinado que pudiera mostrar de forma gráfica las condiciones esperables en cada punto, mostrando, por ejemplo, el fenómeno con mayor impacto para cada punto y su color correspondiente. Se puede ver un ejemplo tentativo en la figura 4.

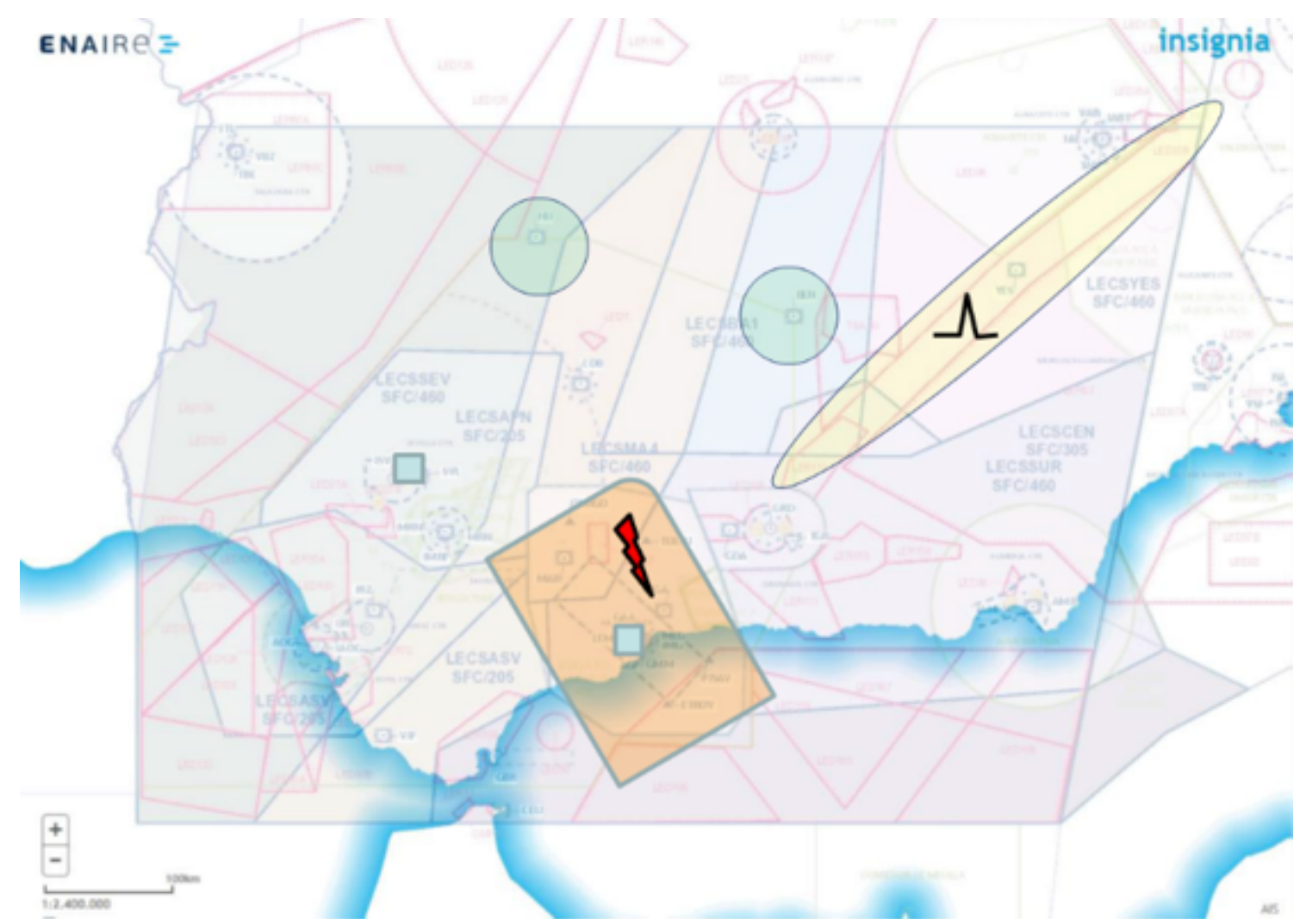

Figura 5. Ejemplo ficticio de producto combinado de impactos meteorológicos.

Lo más importante para un producto de estas características es que se pueda integrar en los protocolos de actuación del proveedor de servicios ATS. Para ello, hay que insistir, es fundamental la definición de umbrales, teniendo en cuenta el lugar, el fenómeno y la hora de ocurrencia (figuras 5 y 6 ). No es fácil y requiere el trabajo conjunto con los responsables del centro de control y los actores de la sala. Por último, para afinar el producto se necesitará un periodo de pruebas que permita la calibración adecuada del mismo.

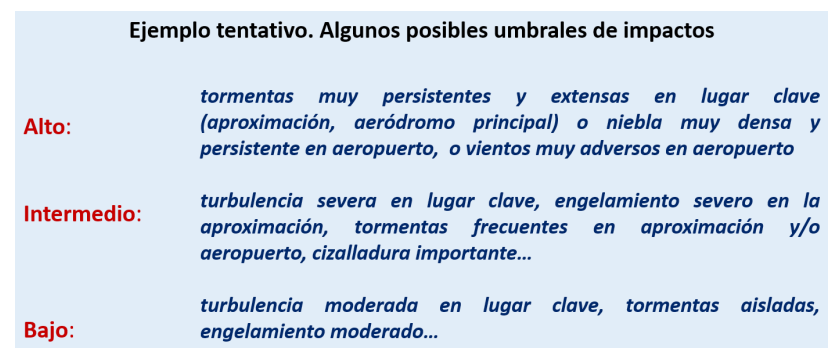

Figura 4. Ejemplo ficticio de definición de umbrales de impacto para distintos fenómenos. 


\subsection{Estado actual. Productos orientados a impacto}

El servicio preoperativo que se presta en el proyecto piloto tiene medios y horarios limitados, que no permiten la puesta en marcha de productos como los descritos en el subapartado anterior. No obstante, se elaboran ya productos orientados a impacto que recogen varias de las características deseables de los futuros productos operativos. En el centro de control de Sevilla se elabora un producto resumen del briefing conteniendo una previsión a 12 horas de fenómenos meteorológicos con impacto en puntos de especial interés (acordados con los responsables del centro de control) y un avance para las siguientes 12 horas. La predicción elaborada tiene carácter probabilístico y es susceptible de enmienda y/o actualización en caso necesario (fig. 7). Con un formato similar se elabora también un avance de previsión e impactos para fines de semana y festivos.

El producto es de gran interés para el usuario, que lo usa para la toma de decisiones operativas. Recientemente se ha utilizado un formato similar para dar apoyo (en este caso sí operativo) a ENAIRE con motivo del ejercicio militar TLP (Tactical Leadership Programme), que tiene implicaciones importantes en el tráfico comercial entre zonas restringidas al tráfico

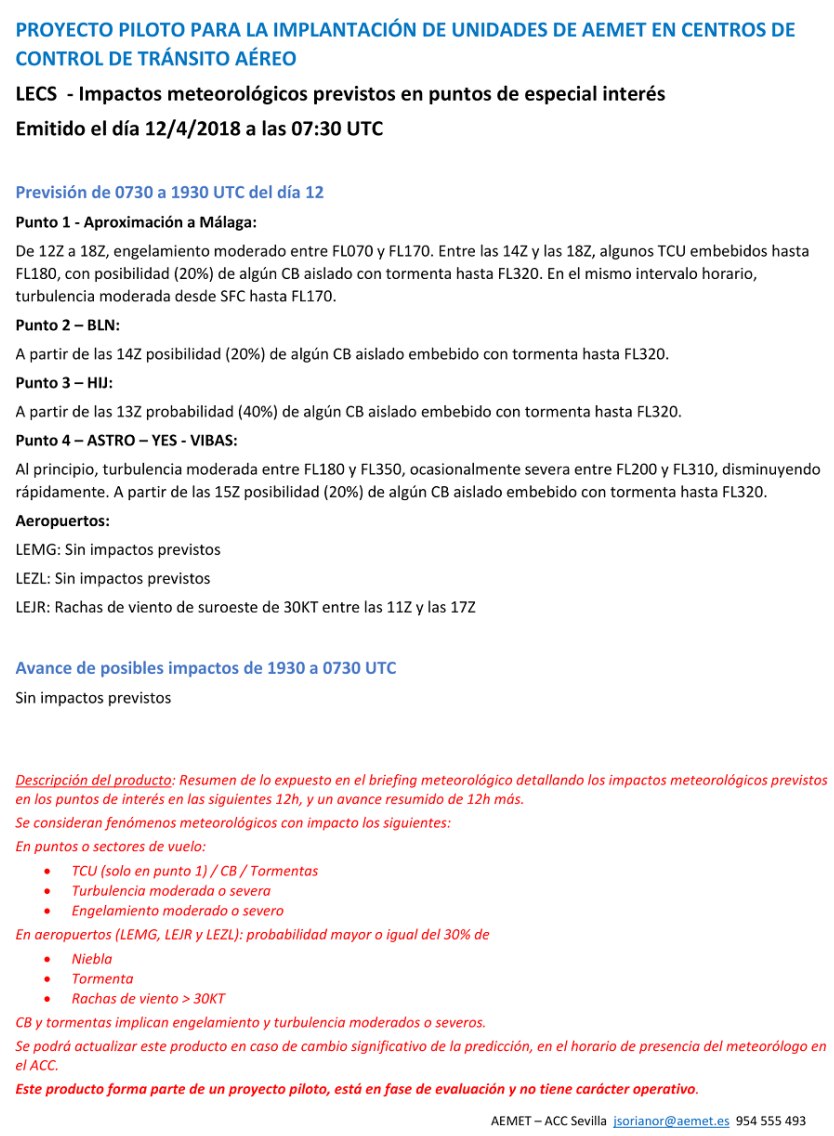

Figura 7. Ejemplo de pronóstico diario orientado a impactos para el centro de control de Sevilla.

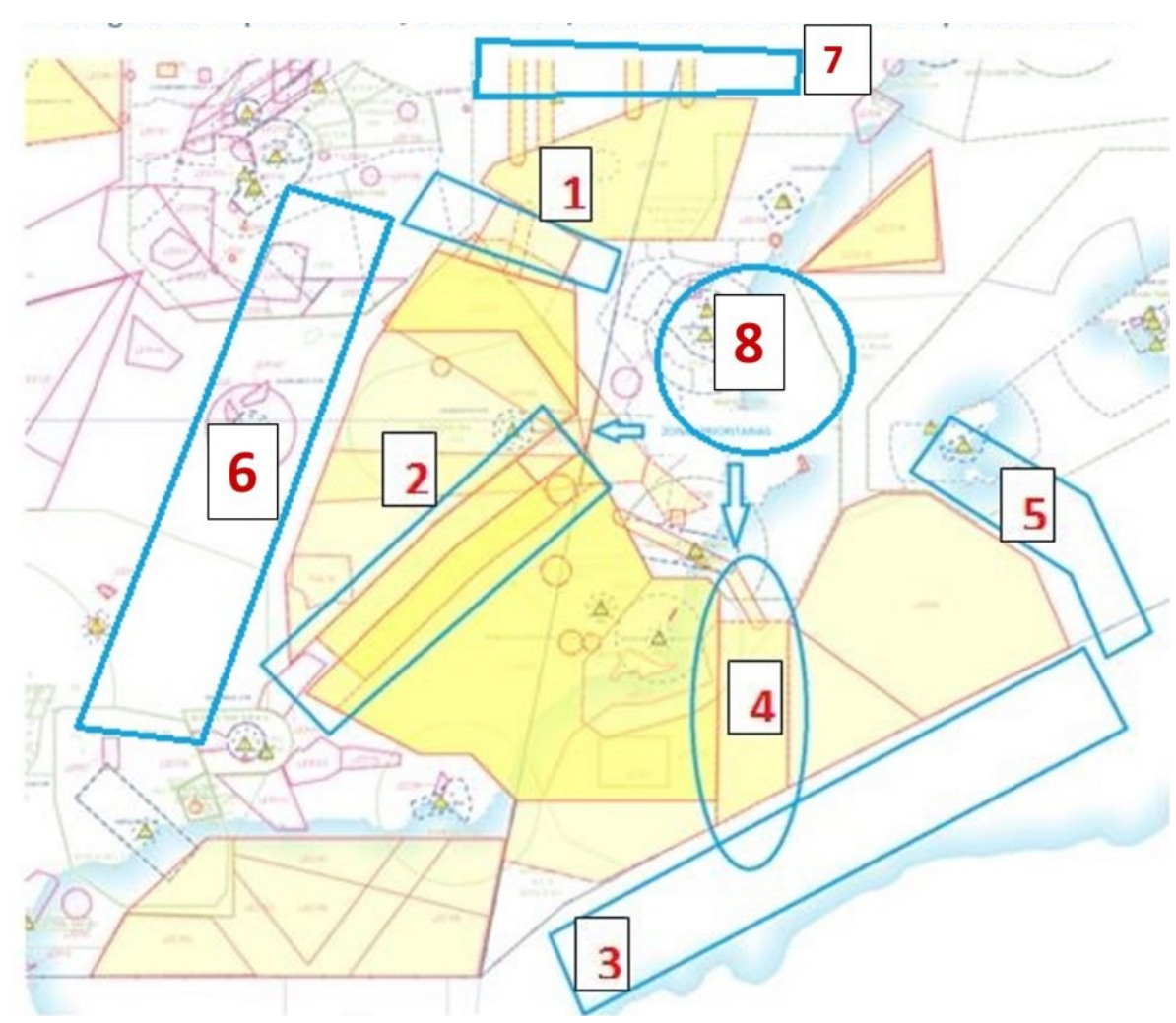

Figura 8. Zonas de especial interés definidas para el ejercicio TLP en septiembre-octubre de 2018. 
militar que ocupan gran parte del espacio aéreo del este y sureste peninsular y sur de Baleares (figura 8). Este último caso es un claro ejemplo de la utilidad de predicciones de impacto de carácter probabilista. Ante una probabilidad media de tormentas en alguno de los pasillos existentes entre las deltas activadas, ENAIRE tomaba la decisión de cerrar completamente al tráfico dicho pasillo, al no ser aceptable por razones de seguridad que los posibles desvíos sobrevenidos provocaran la entrada de aeronaves comerciales en la zona afectada por las maniobras. Un mismo fenómeno con una determinada probabilidad de ocurrencia tiene impactos muy diferentes en función de las características de la zona afectada, la hora del día o las circunstancias del entorno, en este caso, la existencia de un ejercicio militar en los alrededores, y lleva a actuaciones muy diferentes también en un caso u otro.

\section{CONCLUSIONES}

El control del tráfico aéreo tiene el doble objetivo de mantener la seguridad y optimizar los flujos aéreos. Para ello deben tomarse una serie de decisiones operativas que requieren de una información precisa sobre las incidencias que pueden producirse y su impacto en las operaciones. Las incidencias meteorológicas se encuentran entre las más frecuentes e importantes, tanto para la seguridad como para la puntualidad de los vuelos. En un contexto de incremento del tráfico y crecientes exigencias de seguridad y puntualidad, la mejora del apoyo meteorológico se hace aún más necesaria. El catálogo tradicional de productos de meteorología aeronáutica no basta, especialmente a nivel de TMA, donde la complejidad del control aéreo es mayor. Se hace necesario disponer de predicciones orientadas a impacto, que faciliten la toma de decisiones de forma ágil y eficaz.

En ese contexto se ha puesto en marcha un proyecto para implementar unidades de asesoría meteorológica en los centros de control de ENAIRE. Una vez detectadas las necesidades, el servicio preoperativo se estructura en tres actividades principales para dar respuesta a dichas necesidades: asesoría experta, briefing meteorológico y elaboración de pronósticos de impacto.

Cuando las nuevas unidades estén operativas se elaborarán productos basados en matrices de impacto, que tengan en cuenta el nivel de riesgo de los fenómenos meteorológicos con incidencia en la operatividad y su probabilidad de ocurrencia, cuya salida sirva como entrada a los procedimientos de toma de decisiones de los actores del control aéreo. Estos pronósticos deberán elaborarse para puntos y zonas de especial interés con antelación suficiente para que la toma de decisiones sea efectiva. La determinación de los umbrales de los distintos niveles de riesgo y la escala de probabilidades, en estrecha colaboración con ENAIRE, será un punto crucial para que estos productos cumplan con su misión.

Mientras tanto se elaboran ya en la experiencia piloto de presencia de predictores en centros de control predicciones orientadas a impacto en formato texto que tienen en cuenta varios de los requerimientos principales, como la identificación de puntos y zonas de interés, fenómenos con impacto, horario de máxima afluencia de tráficos y disponibilidad con antelación suficiente.

\section{REFERENCIAS}

University Corporation for Atmospheric Research, 2005. The Impact of Weather on Air Traffic Management. Módulo COMET. https://www.meted.ucar.edu/training_module.php?id=168\#.XDWkis39Vpg.

EuROCONTROL, 2013. Severe Weather Risk Management Survey. https://www.eurocontrol.int/sites/default/ files/article/files/severe-weather-risk-management-survey-2013.pdf.

OACI.Anexos. https://store.icao.int/annexes.html. 
ENAIRE. Procedimiento de actuación en caso de meteorología adversa severa por tormentas. Documento interno.

ENAIRE. Cartografía y documentos en web. https://ais.enaire.es/insignia/navegador/.

AEMET. Proyecto para la implantación de unidades de asesoría de AEMET en Centros de Control de ENAIRE. Documentación interna. 